\title{
Novel Piperazinyl-Quinazoline-4-one Analogs: Design, Synthesis and Evaluation of In Vitro Biological Activity
}

\author{
K. N. MYANGAR*, T. N. AKHAJA, D. R. NAIK and J. P. RAVAL \\ Department of Pharmaceutical Chemistry, Ashok \& Rita Patel Institute of Integrated Study \\ and Research in Biotechnology and Allied Sciences (ARIBAS), ADIT Campus, \\ New Vallabh Vidhyanagar - 388 121, Dist: Anand, Gujarat, India \\ kruti_nm@ymail.com
}

Received 8 June 2012 / Accepted 22 June 2012

\begin{abstract}
A series of novel 2-[4-substituted-piperazinyl-methyl]-3-[ $N$-isonicotinamide-yl]-quinazoline4-one 4a-l were designed, synthesized, characterized and evaluated for in vitro antitubercular, antibacterial and antifungal activity. Compounds $4 \mathbf{f}, \mathbf{4 h}$ and $\mathbf{4 l}$ exhibited excellent antitubercular activity against mycobacterium tuberculosis $\mathrm{H}_{37} R v$.
\end{abstract}

Keywords: Piperazine, 2-Chloromethyl-3-( $N$-isonicotinamide-yl)-4Hquinazolinone, Antitubercular activity

\section{Introduction}

Research in heterocyclic chemistry has gained momentum in recent times because more than half of the biologically active molecules belong to various classes of heterocycles ${ }^{1}$, among them quinazolinone have remained always a major source for therapeutic drugs ${ }^{2}$. Also they are the building block for more than 150 naturally occurring pharmacologically active alkaloids and commercial drugs ${ }^{3,4}$.

Structure activity relationship studies of quinazolinone ring system suggest that position 2, 6 and 8 are important for pharmacokinetic property while position 3 attached with different heterocyclic ring system is endowed with better chemotherapeutic activity ${ }^{5}$. On the other hand, piperazine and substituted piperazine are important pharmacophore that can be found in many marketed drugs and drugs under clinical trials ${ }^{6,7}$. Also, piperazines were explored with several biological activity ${ }^{8-11}$. Actually the polarity of nitrogen atoms of piperazine ring enhances favorable interaction with biomacromolecules and thus confers the biological activity ${ }^{12,13}$.

Since these two heterocyclic moieties constitute two active pharmacophore and are supposed to be highly active, combining these two is expected to have a synergistic effect against pathogens causing infectious diseases. Keeping this in mind and to identify new drug candidates, that may be valued in designing new, potent, selective and less toxic anti- 
infective agents and also, our continued interest in the synthesis of novel heterocyclic hybrids ${ }^{14-17}$ with promising antimicrobial activity, herein, we report the synthesis and evaluation of in vitro biological activity of novel piperazinyl-quinazolin-4-one analogs. By applying concept molecular hybridization, we have used introduced antitubercular drug isoniazid at position 3, while at position 2, various substituted piperazine derivatives were attached in quinazolin-4-one heterocycles and tried to get promising anti-infective agents.

\section{Experimental}

The melting points were determined in open glass capillaries and are uncorrected. The purity of all the newly synthesized compounds were routinely checked by TLC ( $0.5 \mathrm{~mm}$ thickness) using silica gel-G coated aluminium plates (Merck) and spots were visualized by exposing the dry plates in iodine vapors. IR spectra $\left(v_{\max }\right.$ in $\left.\mathrm{cm}^{-1}\right)$ were recorded on a Perkin-Elmer 1720 FT-IR spectrometer ( $\mathrm{KBr}$ pellets). ${ }^{1} \mathrm{H}-\mathrm{NMR}$ and ${ }^{13} \mathrm{C}-\mathrm{NMR}$ spectra were recorded on a Bruker AC $400 \mathrm{MHz}$ and $100 \mathrm{MHz}$ spectrometer respectively in DMSO- $d_{6}$, referenced to TMS. Mass spectra were recorded on Shimadzu LCMS 2010 spectrometer. Elemental analyses $(\mathrm{C}, \mathrm{H}$, and $\mathrm{N})$ were conducted using a Carlo Erba analyzer model 1106.

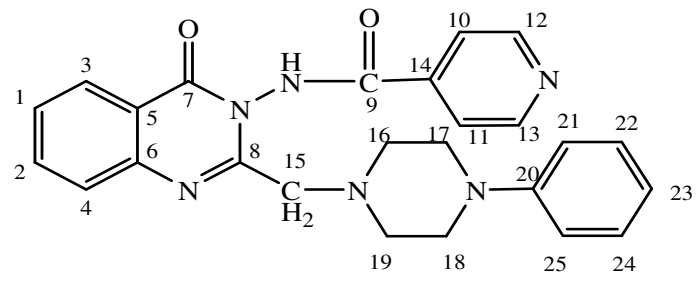

Figure 1. Numbering system for 13C-NMR of compounds $\mathbf{4 a}, \mathbf{4 c - g}$ and $\mathbf{4 i - 1}$

General procedure for the synthesis of 2-[4-substituted-piperazinyl-methyl]-3-[Nisonicotinamide-yl]-quinazoline-4-one (4a-l)

A mixture of compound $\mathbf{3 a} / \mathbf{3 b}(0.5 \mathrm{mmol})$, appropriate piperazine $(0.7 \mathrm{mmol})$ and anhydrous sodium carbonate $(1.50 \mathrm{~g})$ in absolute ethanol was refluxed for $8-9 \mathrm{~h}$ (reaction progress was monitored on TLC). After completion, the excess of amine and ethanol was removed by distillation and the residue was treated with $5 \% \mathrm{NaHCO}_{3}$ solution to remove acidic impurities, filtered, washed and dried. Final products were crystallized using ethanol to give the title compounds.

\section{2-[4-Phenyl-piperazinyl-methyl]-3-[N-isonicotinamide-yl]-quinazoline-4-one (4a)}

Yield, 68\%; m.p. 179-181 ${ }^{\circ} \mathrm{C}$; FTIR (KBr) v: 3368 (NH), 3036 (C-H Aromatic), 2954 (C-H aliphatic), $1678(\mathrm{C}=\mathrm{O}$ of amide), $1642(\mathrm{C}=\mathrm{O}$ of quinazolinone $), 1352(\mathrm{C}=\mathrm{N}), 1290(\mathrm{C}-\mathrm{N})$, $1226(\mathrm{~N}-\mathrm{N}) \mathrm{cm}^{-1} ;{ }^{1} \mathrm{H}$ NMR $\left(400 \mathrm{MHz}, \mathrm{DMSO}-d_{6}\right) \delta \mathrm{ppm}: 1.82\left(\mathrm{~s}, 2 \mathrm{H}, \mathrm{CH}_{2}\right), 2.48-2.65$ $\left(\mathrm{m}, 8 \mathrm{H}, 4 \times \mathrm{CH}_{2}\right), 6.73-8.28(\mathrm{~m}, 13 \mathrm{H}, \mathrm{Ar}-\mathrm{H}), 8.54$ (s, $\left.1 \mathrm{H}, \mathrm{NH}-\mathrm{CO}\right) ;{ }^{13} \mathrm{C} \mathrm{NMR}(100 \mathrm{MHz}$, DMSO- $\left.d_{6}\right) \delta$ ppm: $51.22\left(\mathrm{C}_{15}\right), 55.30\left(\mathrm{C}_{16}, \mathrm{C}_{19}\right), 57.43\left(\mathrm{C}_{17}, \mathrm{C}_{18}\right), 122.48-133.27\left(\mathrm{C}_{1}-\mathrm{C}_{5}, \mathrm{C}_{10^{-}}\right.$ $\left.\mathrm{C}_{11}, \mathrm{C}_{20}-\mathrm{C}_{25}\right), 142.18\left(\mathrm{C}_{14}\right), 147.72\left(\mathrm{C}_{6}\right), 150.44\left(\mathrm{C}_{12}-\mathrm{C}_{13}\right), 164.21\left(\mathrm{C}_{8}\right), 167.20\left(\mathrm{C}_{7}\right), 168.34$ $\left(\mathrm{C}_{9}\right)$; MS: $m / z$ [440.20] $]^{+}$; Analysis calculated for $\mathrm{C}_{25} \mathrm{H}_{24} \mathrm{~N}_{6} \mathrm{O}_{2}: \mathrm{C}, 68.17 ; \mathrm{H}, 5.49 ; \mathrm{N}, 19.08$. Found: C, 68.32; H, 5.66; N, 19.23\%.

\section{2-[4-Benzyl-piperazinyl-methyl]-3-[N-isonicotinamide-yl]-quinazoline-4-one (4b)}

Yield, 72\%; m.p. 198-200 ${ }^{\circ} \mathrm{C}$; FTIR (KBr) v: 3379 (NH), 3048 (C-H Aromatic), 2959 (C-H aliphatic), $1682(\mathrm{C}=\mathrm{O}$ of amide), $1644(\mathrm{C}=\mathrm{O}$ of quinazolinone), $1360(\mathrm{C}=\mathrm{N}), 1283$ $(\mathrm{C}-\mathrm{N}), 1230(\mathrm{~N}-\mathrm{N}) \mathrm{cm}^{-1}$; ${ }^{1} \mathrm{H}$ NMR (400MHz, DMSO-d $\left.d_{6}\right) \delta \mathrm{ppm}: 1.74\left(\mathrm{~s}, 2 \mathrm{H}, \mathrm{CH}_{2}\right), 2.34-2.46$ 
$\left(\mathrm{m}, 8 \mathrm{H}, 4 \times \mathrm{CH}_{2}\right), 2.60\left(\mathrm{~s}, 2 \mathrm{H}, \mathrm{CH}_{2}\right), 7.32-8.12(\mathrm{~m}, 13 \mathrm{H}, \mathrm{Ar}-\mathrm{H}), 8.67$ (s, 1H, NH-CO); ${ }^{13} \mathrm{C}$ NMR $\left(100 \mathrm{MHz}\right.$, DMSO- $\left.d_{6}\right) \delta \mathrm{ppm}$ : $51.28\left(\mathrm{C}_{15}\right), 55.48\left(\mathrm{C}_{16}, \mathrm{C}_{19}\right), 57.32\left(\mathrm{C}_{17}, \mathrm{C}_{18}\right), 60.23$ $\left(\mathrm{CH}_{2}\right), 122.32-136.27\left(\mathrm{C}_{1}-\mathrm{C}_{5}, \mathrm{C}_{10}-\mathrm{C}_{11}, \mathrm{C}_{20}-\mathrm{C}_{25}\right), 142.20\left(\mathrm{C}_{14}\right), 147.63\left(\mathrm{C}_{6}\right), 150.39\left(\mathrm{C}_{12}-\mathrm{C}_{13}\right)$, $164.14\left(\mathrm{C}_{8}\right), 167.23\left(\mathrm{C}_{7}\right), 168.40\left(\mathrm{C}_{9}\right)$; MS: $\mathrm{m} / \mathrm{z}[454.21]^{+}$; Analysis calculated for $\mathrm{C}_{26} \mathrm{H}_{26} \mathrm{~N}_{6} \mathrm{O}_{2}$ : C, 68.70; H, 5.77; N, 18.49. Found: C, 68.82; H, 5.59; N, 18.34\%.

\section{2-[4-Methyl-piperazinyl-methyl]-3-[N-isonicotinamide-yl]-quinazoline-4-one (4c)}

Yield, 70\%; m.p. 176-178 ${ }^{\circ} \mathrm{C}$; FTIR (KBr) v: 3358 (NH), 3053 (C-H Aromatic), 2947 (C-H aliphatic), $1673(\mathrm{C}=\mathrm{O}$ of amide), $1631(\mathrm{C}=\mathrm{O}$ of quinazolinone $), 1367(\mathrm{C}=\mathrm{N}), 1278(\mathrm{C}-\mathrm{N})$, $1224(\mathrm{~N}-\mathrm{N}) \mathrm{cm}^{-1} ;{ }^{1} \mathrm{H}$ NMR $\left(400 \mathrm{MHz}, \mathrm{DMSO}-d_{6}\right) \delta \mathrm{ppm}$ : $1.81\left(\mathrm{~s}, 2 \mathrm{H}, \mathrm{CH}_{2}\right), 2.32(\mathrm{~s}, 3 \mathrm{H}$, $\left.\mathrm{CH}_{3}\right), 2.49-2.67$ (m, 8H, $\left.4 \times \mathrm{CH}_{2}\right), 7.27-8.28(\mathrm{~m}, 8 \mathrm{H}, \mathrm{Ar}-\mathrm{H}), 8.70$ (s, $\left.1 \mathrm{H}, \mathrm{NH}-\mathrm{CO}\right) ;{ }^{13} \mathrm{C} \mathrm{NMR}$ $\left(100 \mathrm{MHz}, \mathrm{DMSO}-d_{6}\right) \delta \mathrm{ppm}: 38.62\left(\mathrm{CH}_{3}\right), 51.35\left(\mathrm{C}_{15}\right), 55.18\left(\mathrm{C}_{16}, \mathrm{C}_{19}\right), 57.24\left(\mathrm{C}_{17}, \mathrm{C}_{18}\right)$, 122.24-133.38 $\left(\mathrm{C}_{1}-\mathrm{C}_{5}, \mathrm{C}_{10}-\mathrm{C}_{11}\right), 142.34\left(\mathrm{C}_{14}\right), 147.58\left(\mathrm{C}_{6}\right), 150.43\left(\mathrm{C}_{12}-\mathrm{C}_{13}\right), 164.21\left(\mathrm{C}_{8}\right)$, $167.30\left(\mathrm{C}_{7}\right), 168.44\left(\mathrm{C}_{9}\right)$; MS: $m / z$ [378.18] $]^{+}$; Analysis calculated for $\mathrm{C}_{20} \mathrm{H}_{22} \mathrm{~N}_{6} \mathrm{O}_{2}: \mathrm{C}, 63.48$; H, 5.86; N, 22.21. Found: C, 63.34; H, 5.75; N, 22.40\%.

\section{2-[4-(2-Methoxy-phenyl)-piperazinyl-methyl]-3-[N-isonicotinamide-yl]-quinazoline -4-one (4d)}

Yield, 68\%; m.p. 223-225 ${ }^{\circ} \mathrm{C}$; FTIR (KBr) v: $3362(\mathrm{NH}), 3049$ (C-H Aromatic), 2956 (C-H aliphatic), $1680(\mathrm{C}=\mathrm{O}$ of amide $), 1638(\mathrm{C}=\mathrm{O}$ of quinazolinone $), 1372(\mathrm{C}=\mathrm{N}), 1294(\mathrm{C}-\mathrm{N})$, $1218(\mathrm{~N}-\mathrm{N}) \mathrm{cm}^{-1} ;{ }^{1} \mathrm{H}$ NMR $\left(400 \mathrm{MHz}, \mathrm{DMSO}-d_{6}\right) \delta \mathrm{ppm}: 1.92\left(\mathrm{~s}, 2 \mathrm{H}, \mathrm{CH}_{2}\right), 2.36-2.58(\mathrm{~m}, 8 \mathrm{H}$, $\left.4 \times \mathrm{CH}_{2}\right), 3.88\left(\mathrm{~s}, 2 \mathrm{H}, \mathrm{OCH}_{3}\right), 6.57-8.06(\mathrm{~m}, 12 \mathrm{H}, \mathrm{Ar}-\mathrm{H}), 8.73(\mathrm{~s}, 1 \mathrm{H}, \mathrm{NH}-\mathrm{CO}) ;{ }^{13} \mathrm{C} \mathrm{NMR}$ $\left(100 \mathrm{MHz}, \mathrm{DMSO}-d_{6}\right) \delta$ ppm: $51.29\left(\mathrm{C}_{15}\right), 55.19\left(\mathrm{C}_{16}, \mathrm{C}_{19}\right), 56.27\left(\mathrm{OCH}_{3}\right), 57.83\left(\mathrm{C}_{17}, \mathrm{C}_{18}\right)$, 115.89-133.13 $\left(\mathrm{C}_{1}-\mathrm{C}_{5}, \mathrm{C}_{10}-\mathrm{C}_{11}, \mathrm{C}_{20}-\mathrm{C}_{23}, \mathrm{C}_{25}\right), 142.26\left(\mathrm{C}_{14}\right), 146.52\left(\mathrm{C}_{24}\right), 147.75\left(\mathrm{C}_{6}\right), 150.23$ $\left(\mathrm{C}_{12}-\mathrm{C}_{13}\right), 164.20\left(\mathrm{C}_{8}\right), 167.25\left(\mathrm{C}_{7}\right), 168.49\left(\mathrm{C}_{9}\right) ; \mathrm{MS}: \mathrm{m} / z$ [470.21] $]^{+}$; Analysis calculated for $\mathrm{C}_{26} \mathrm{H}_{26} \mathrm{~N}_{6} \mathrm{O}_{3}$ : C, 66.37; H, 5.57; N, 17.86. Found: C, 66.48; H, 5.42; N, 17.93\%.

2-[4-(4-Methoxy-phenyl)-piperazinyl-methyl]-3-[N-isonicotinamide-yl]-quinazoline -4-one (4e)

Yield, 67\%; m.p. 236-238 ${ }^{\circ} \mathrm{C}$; FTIR (KBr) v: 3357 (NH), 3061 (C-H Aromatic), 2948 (C-H aliphatic), $1674(\mathrm{C}=\mathrm{O}$ of amide), $1623(\mathrm{C}=\mathrm{O}$ of quinazolinone), $1365(\mathrm{C}=\mathrm{N}), 1305(\mathrm{C}-\mathrm{N})$, $1226(\mathrm{~N}-\mathrm{N}) \mathrm{cm}^{-1} ;{ }^{1} \mathrm{H}$ NMR $\left(400 \mathrm{MHz}, \mathrm{DMSO}-d_{6}\right) \delta \mathrm{ppm}: 1.84\left(\mathrm{~s}, 2 \mathrm{H}, \mathrm{CH}_{2}\right), 2.40-2.64(\mathrm{~m}, 8 \mathrm{H}$, $\left.4 \times \mathrm{CH}_{2}\right), 3.90\left(\mathrm{~s}, 2 \mathrm{H}, \mathrm{OCH}_{3}\right), 6.71-8.13(\mathrm{~m}, 12 \mathrm{H}, \mathrm{Ar}-\mathrm{H}), 8.79(\mathrm{~s}, 1 \mathrm{H}, \mathrm{NH}-\mathrm{CO}) ;{ }^{13} \mathrm{C} \mathrm{NMR}$ $\left(100 \mathrm{MHz}, \mathrm{DMSO}-d_{6}\right) \delta$ ppm: $51.38\left(\mathrm{C}_{15}\right), 55.30\left(\mathrm{C}_{16}, \mathrm{C}_{19}\right), 56.15\left(\mathrm{OCH}_{3}\right), 57.78\left(\mathrm{C}_{17}, \mathrm{C}_{18}\right)$, 115.94-136.13 $\left(\mathrm{C}_{1}-\mathrm{C}_{5}, \mathrm{C}_{10}-\mathrm{C}_{11}, \mathrm{C}_{20}-\mathrm{C}_{21}, \mathrm{C}_{23}-\mathrm{C}_{25}\right), 142.35\left(\mathrm{C}_{14}\right), 147.82\left(\mathrm{C}_{6}\right), 150.18\left(\mathrm{C}_{12}-\mathrm{C}_{13}\right)$, $151.43\left(\mathrm{C}_{22}\right), 164.16\left(\mathrm{C}_{8}\right), 167.30\left(\mathrm{C}_{7}\right), 168.53\left(\mathrm{C}_{9}\right)$; MS: $\mathrm{m} / \mathrm{z}[470.21]^{+}$; Analysis calculated for $\mathrm{C}_{26} \mathrm{H}_{26} \mathrm{~N}_{6} \mathrm{O}_{3}$ : C, 66.37; H, 5.57; N, 17.86. Found: C, 66.28; H, 5.76; N, 17.77\%.

2-[4-(2,3-Dichloro-phenyl)-piperazinyl-methyl]-3-[N-isonicotinamide-yl]quinazoline 4-one (4f)

Yield, 73\%; m.p. 219-221 ${ }^{\circ} \mathrm{C}$; FTIR (KBr) v: 3364 (NH), 3047 (C-H Aromatic), 2953 (C-H aliphatic), $1682(\mathrm{C}=\mathrm{O}$ of amide), $1630(\mathrm{C}=\mathrm{O}$ of quinazolinone $), 1357(\mathrm{C}=\mathrm{N}), 1291(\mathrm{C}-\mathrm{N})$, $1230(\mathrm{~N}-\mathrm{N}) \mathrm{cm}^{-1}$; ${ }^{1} \mathrm{H}$ NMR $\left(400 \mathrm{MHz}, \mathrm{DMSO}-d_{6}\right) \delta \mathrm{ppm}: 1.98\left(\mathrm{~s}, 2 \mathrm{H}, \mathrm{CH}_{2}\right), 2.39-2.68(\mathrm{~m}$, $\left.8 \mathrm{H}, 4 \times \mathrm{CH}_{2}\right), 6.57-8.10(\mathrm{~m}, 11 \mathrm{H}, \mathrm{Ar}-\mathrm{H}), 8.84(\mathrm{~s}, 1 \mathrm{H}, \mathrm{NH}-\mathrm{CO}) ;{ }^{13} \mathrm{C}$ NMR $(100 \mathrm{MHz}$, DMSO$\left.d_{6}\right) \delta$ ppm: $51.48\left(\mathrm{C}_{15}\right), 55.36\left(\mathrm{C}_{16}, \mathrm{C}_{19}\right), 57.25\left(\mathrm{C}_{17}, \mathrm{C}_{18}\right), 116.79-135.81\left(\mathrm{C}_{1}-\mathrm{C}_{5}, \mathrm{C}_{10}-\mathrm{C}_{11}\right.$, $\left.\mathrm{C}_{20}-\mathrm{C}_{24}\right), 142.23\left(\mathrm{C}_{14}\right), 146.48\left(\mathrm{C}_{25}\right), 147.78\left(\mathrm{C}_{6}\right), 150.22\left(\mathrm{C}_{12}-\mathrm{C}_{13}\right), 164.21\left(\mathrm{C}_{8}\right), 167.28\left(\mathrm{C}_{7}\right)$, 168.45 $\left(\mathrm{C}_{9}\right)$; MS: $\mathrm{m} / z$ [508.12] $]^{+}$; Analysis calculated for $\mathrm{C}_{25} \mathrm{H}_{22} \mathrm{Cl}_{2} \mathrm{~N}_{6} \mathrm{O}_{2}$ : C, 58.95; $\mathrm{H}, 4.35$; $\mathrm{N}, 16.50$. Found: C, 58.80; H, 4.56; N, 16.37\%. 
2-[4-Phenyl-piperazinyl-methyl]-3-[N-isonicotinamide-yl]-6-iodo-quinazoline-4one (4g)

Yield, 69\%; m.p. 217-219 ${ }^{\circ} \mathrm{C}$; FTIR (KBr) v: $3350(\mathrm{NH}), 3048$ (C-H Aromatic), 2952 (C-H aliphatic), $1684(\mathrm{C}=\mathrm{O}$ of amide), $1623(\mathrm{C}=\mathrm{O}$ of quinazolinone $), 1360(\mathrm{C}=\mathrm{N}), 1290(\mathrm{C}-\mathrm{N})$, $1231(\mathrm{~N}-\mathrm{N}), 552(\mathrm{C}-\mathrm{I}) \mathrm{cm}^{-1} ;{ }^{1} \mathrm{H}$ NMR $\left(400 \mathrm{MHz}, \mathrm{DMSO}-d_{6}\right) \delta$ ppm: $1.75\left(\mathrm{~s}, 2 \mathrm{H}, \mathrm{CH}_{2}\right), 2.50-$ $2.76\left(\mathrm{~m}, 8 \mathrm{H}, 4 \times \mathrm{CH}_{2}\right), 6.51-8.28(\mathrm{~m}, 12 \mathrm{H}, \mathrm{Ar}-\mathrm{H}), 8.63$ (s, $\left.1 \mathrm{H}, \mathrm{NH}-\mathrm{CO}\right) ;{ }^{13} \mathrm{C} \mathrm{NMR}(100 \mathrm{MHz}$, DMSO- $\left.d_{6}\right) \delta$ ppm: $51.17\left(\mathrm{C}_{15}\right), 55.31\left(\mathrm{C}_{16}, \mathrm{C}_{19}\right), 57.52\left(\mathrm{C}_{17}, \mathrm{C}_{18}\right), 95.80\left(\mathrm{C}_{1}\right), 122.51-137.38$ $\left(\mathrm{C}_{3}-\mathrm{C}_{5}, \mathrm{C}_{10}-\mathrm{C}_{11}, \mathrm{C}_{20}-\mathrm{C}_{24}\right), 142.17\left(\mathrm{C}_{2}\right), 142.54\left(\mathrm{C}_{14}\right), 144.40\left(\mathrm{C}_{25}\right), 147.49\left(\mathrm{C}_{6}\right), 150.42$ $\left(\mathrm{C}_{12}-\mathrm{C}_{13}\right), 164.19\left(\mathrm{C}_{8}\right), 167.21\left(\mathrm{C}_{7}\right), 168.46\left(\mathrm{C}_{9}\right)$; MS: $\mathrm{m} / z$ [566.09] ${ }^{+}$; Analysis calculated for $\mathrm{C}_{25} \mathrm{H}_{23} \mathrm{IN}_{6} \mathrm{O}_{2}$ : C, 53.01; H, 4.09; N, 14.84. Found: C, 53.13; H, 4.20; N, 14.72\%.

\section{2-[4-Benzyl-piperazinyl-methyl]-3-[N-isonicotinamide-yl]-6-iodo-quinazoline-4-one}

\section{(4h)}

Yield, 71\%; m.p. 209-211 ${ }^{\circ} \mathrm{C}$; FTIR (KBr) v: 3367 (NH), 3072 (C-H Aromatic), 2946 (C-H aliphatic), $1688(\mathrm{C}=\mathrm{O}$ of amide $), 1628(\mathrm{C}=\mathrm{O}$ of quinazolinone $), 1364(\mathrm{C}=\mathrm{N}), 1284(\mathrm{C}-\mathrm{N})$, $1220(\mathrm{~N}-\mathrm{N}), 542(\mathrm{C}-\mathrm{I}) \mathrm{cm}^{-1}$; ${ }^{1} \mathrm{H}$ NMR $\left(400 \mathrm{MHz}, \mathrm{DMSO}-d_{6}\right) \delta \mathrm{ppm}: 1.85\left(\mathrm{~s}, 2 \mathrm{H}, \mathrm{CH}_{2}\right), 2.35-$ $2.68\left(\mathrm{~m}, 8 \mathrm{H}, 4 \times \mathrm{CH}_{2}\right), 3.58\left(\mathrm{~s}, 2 \mathrm{H}, \mathrm{CH}_{2}\right), 7.34-8.19$ (m, 12H, Ar-H), 8.59 (s, 1H, NH-CO); ${ }^{13} \mathrm{C}-\mathrm{NMR}\left(100 \mathrm{MHz}, \mathrm{DMSO}-d_{6}\right) \delta \mathrm{ppm}: 51.17\left(\mathrm{C}_{15}\right), 55.53\left(\mathrm{C}_{16}, \mathrm{C}_{19}\right), 57.24\left(\mathrm{C}_{17}, \mathrm{C}_{18}\right), 60.12$ $\left(\mathrm{CH}_{2}\right), 95.74\left(\mathrm{C}_{1}\right), 122.26-136.30\left(\mathrm{C}_{3}-\mathrm{C}_{5}, \mathrm{C}_{10}-\mathrm{C}_{11}, \mathrm{C}_{20}-\mathrm{C}_{25}\right), 142.11\left(\mathrm{C}_{2}\right), 142.48\left(\mathrm{C}_{14}\right), 147.51$ $\left(\mathrm{C}_{6}\right), 150.33\left(\mathrm{C}_{12}-\mathrm{C}_{13}\right), 164.16\left(\mathrm{C}_{8}\right), 167.25\left(\mathrm{C}_{7}\right), 168.35\left(\mathrm{C}_{9}\right)$; MS: $\mathrm{m} / z$ [580.11] ${ }^{+}$; Analysis calculated for $\mathrm{C}_{26} \mathrm{H}_{25} \mathrm{IN}_{6} \mathrm{O}_{2}$ : C, 53.80; H, 4.34; N, 14.48. Found: C, 53.87; H, 4.45; N, $14.32 \%$.

2-[4-Methyl-piperazinyl-methyl]-3-[N-isonicotinamide-yl]-6-iodo-quinazoline-4one (4i)

Yield, 68\%; m.p. 189-191 ${ }^{\circ} \mathrm{C}$; FTIR (KBr) v: 3354 (NH), 3064 (C-H Aromatic), 2956 (C-H aliphatic), $1677(\mathrm{C}=\mathrm{O}$ of amide), $1635(\mathrm{C}=\mathrm{O}$ of quinazolinone), $1358(\mathrm{C}=\mathrm{N}), 1271(\mathrm{C}-\mathrm{N})$, $1226(\mathrm{~N}-\mathrm{N}), 538(\mathrm{C}-\mathrm{I}) \mathrm{cm}^{-1}$; ${ }^{1} \mathrm{H}$ NMR $\left(400 \mathrm{MHz}, \mathrm{DMSO}-d_{6}\right) \delta \mathrm{ppm}: 1.76\left(\mathrm{~s}, 2 \mathrm{H}, \mathrm{CH}_{2}\right), 2.18(\mathrm{~s}$, $\left.3 \mathrm{H}, \mathrm{CH}_{3}\right), 2.46-2.70\left(\mathrm{~m}, 8 \mathrm{H}, 4 \times \mathrm{CH}_{2}\right), 7.23-8.30(\mathrm{~m}, 7 \mathrm{H}, \mathrm{Ar}-\mathrm{H}), 8.74(\mathrm{~s}, 1 \mathrm{H}, \mathrm{NH}-\mathrm{CO})$; ${ }^{13} \mathrm{C}-\mathrm{NMR}\left(100 \mathrm{MHz}, \mathrm{DMSO}-d_{6}\right) \delta \mathrm{ppm}: 38.36\left(\mathrm{CH}_{3}\right), 51.43\left(\mathrm{C}_{15}\right), 55.26\left(\mathrm{C}_{16}, \mathrm{C}_{19}\right), 57.38\left(\mathrm{C}_{17}\right.$, $\left.\mathrm{C}_{18}\right)$, $95.67\left(\mathrm{C}_{1}\right), 122.13-137.13\left(\mathrm{C}_{3}-\mathrm{C}_{5}, \mathrm{C}_{10}-\mathrm{C}_{11}\right), 142.14\left(\mathrm{C}_{2}\right), 142.52\left(\mathrm{C}_{14}\right), 147.60\left(\mathrm{C}_{6}\right)$, $150.38\left(\mathrm{C}_{12}-\mathrm{C}_{13}\right), 164.15\left(\mathrm{C}_{8}\right), 167.37\left(\mathrm{C}_{7}\right), 168.51\left(\mathrm{C}_{9}\right) ; \mathrm{MS}: \mathrm{m} / \mathrm{z}[504.08]^{+}$; Analysis calculated for $\mathrm{C}_{20} \mathrm{H}_{21} \mathrm{IN}_{6} \mathrm{O}_{2}$ : C, 47.63; H, 4.20; N, 16.66. Found: C, 47.56; H, 4.27; N, 16.54\%.

2-[4-(2-Methoxy-phenyl)-piperazinyl-methyl]-3-[N-isonicotinamide-yl]-6-iodo-quinazoline-4-one $(\mathbf{4 j})$

Yield, 70\%; m.p. 234-236 ${ }^{\circ} \mathrm{C}$; FTIR (KBr) v: 3336 (NH), 3065 (C-H Aromatic), 2924 (C-H aliphatic), $1697(\mathrm{C}=\mathrm{O}$ of amide), $1630(\mathrm{C}=\mathrm{O}$ of quinazolinone), $1324(\mathrm{C}=\mathrm{N}), 1283(\mathrm{C}-\mathrm{N}), 1247$ $(\mathrm{N}-\mathrm{N}), 542(\mathrm{C}-\mathrm{I}) \mathrm{cm}^{-1}$; ${ }^{1} \mathrm{H}$ NMR (400MHz, DMSO- $\left.d_{6}\right) \delta \mathrm{ppm}: 1.86\left(\mathrm{~s}, 2 \mathrm{H}, \mathrm{CH}_{2}\right), 2.36-2.63$ $\left(\mathrm{m}, 8 \mathrm{H}, 4 \times \mathrm{CH}_{2}\right), 3.79\left(\mathrm{~s}, 2 \mathrm{H}, \mathrm{OCH}_{3}\right), 6.52-8.16(\mathrm{~m}, 11 \mathrm{H}, \mathrm{Ar}-\mathrm{H}), 8.68(\mathrm{~s}, 1 \mathrm{H}, \mathrm{NH}-\mathrm{CO}) ;{ }^{13} \mathrm{C} \mathrm{NMR}$ $\left(100 \mathrm{MHz}, \mathrm{DMSO}-d_{6}\right) \delta$ ppm: $51.34\left(\mathrm{C}_{15}\right), 55.30\left(\mathrm{C}_{16}, \mathrm{C}_{19}\right), 56.15\left(\mathrm{OCH}_{3}\right), 57.80\left(\mathrm{C}_{17}, \mathrm{C}_{18}\right), 95.53$ $\left(\mathrm{C}_{1}\right), 122.18-137.23\left(\mathrm{C}_{3}-\mathrm{C}_{5}, \mathrm{C}_{10}-\mathrm{C}_{11}, \mathrm{C}_{20}-\mathrm{C}_{23}, \mathrm{C}_{25}\right), 142.17\left(\mathrm{C}_{2}\right), 142.48\left(\mathrm{C}_{14}\right), 146.59\left(\mathrm{C}_{24}\right), 147.64$ $\left(\mathrm{C}_{6}\right), 150.14\left(\mathrm{C}_{12}-\mathrm{C}_{13}\right), 164.18\left(\mathrm{C}_{8}\right), 167.31\left(\mathrm{C}_{7}\right), 168.53\left(\mathrm{C}_{9}\right)$; MS: $\mathrm{m} / \mathrm{z}$ [596.10] ${ }^{+}$; Analysis calculated for $\mathrm{C}_{26} \mathrm{H}_{25} \mathrm{IN}_{6} \mathrm{O}_{3}: \mathrm{C}, 52.36 ; \mathrm{H}, 4.22 ; \mathrm{N}, 14.09$. Found: $\mathrm{C}, 52.45 ; \mathrm{H}, 4.36 ; \mathrm{N}, 14.13 \%$.

2-[4-(4-Methoxy-phenyl)-piperazinyl-methyl]-3-[N-isonicotinamide-yl]-6-iodoquinazoline-4-one (4k)

Yield, 71\%; m.p. 243-245 ${ }^{\circ} \mathrm{C}$; FTIR (KBr) v: 3348 (NH), 3054 (C-H Aromatic), 2932 (C-H aliphatic), $1684(\mathrm{C}=\mathrm{O}$ of amide), $1637(\mathrm{C}=\mathrm{O}$ of quinazolinone $), 1316(\mathrm{C}=\mathrm{N}), 1276(\mathrm{C}-\mathrm{N})$, 
$1253(\mathrm{~N}-\mathrm{N}), 531(\mathrm{C}-\mathrm{I}) \mathrm{cm}^{-1} ;{ }^{1} \mathrm{H}$ NMR (400MHz, DMSO- $\left.d_{6}\right) \delta \mathrm{ppm}: 1.78$ (s, 2H, $\mathrm{CH}_{2}$ ), 2.32-2.53 $\left(\mathrm{m}, 8 \mathrm{H}, 4 \times \mathrm{CH}_{2}\right), 3.86\left(\mathrm{~s}, 2 \mathrm{H}, \mathrm{OCH}_{3}\right), 6.68-8.16(\mathrm{~m}, 11 \mathrm{H}, \mathrm{Ar}-\mathrm{H}), 8.64(\mathrm{~s}, 1 \mathrm{H}, \mathrm{NH}-\mathrm{CO}) ;{ }^{13} \mathrm{C} \mathrm{NMR}$ $\left(100 \mathrm{MHz}, \mathrm{DMSO}-d_{6}\right) \delta$ ppm: $51.43\left(\mathrm{C}_{15}\right), 55.26\left(\mathrm{C}_{16}, \mathrm{C}_{19}\right), 56.21\left(\mathrm{OCH}_{3}\right), 57.70\left(\mathrm{C}_{17}, \mathrm{C}_{18}\right), 95.44$ $\left(\mathrm{C}_{1}\right), 122.23-137.34\left(\mathrm{C}_{3}-\mathrm{C}_{5}, \mathrm{C}_{10}-\mathrm{C}_{11}, \mathrm{C}_{20}-\mathrm{C}_{21}, \mathrm{C}_{23}-\mathrm{C}_{25}\right), 142.23\left(\mathrm{C}_{2}\right), 142.56\left(\mathrm{C}_{14}\right), 147.87\left(\mathrm{C}_{6}\right), 150.20$ $\left(\mathrm{C}_{12}-\mathrm{C}_{13}\right), 151.52\left(\mathrm{C}_{22}\right), 164.13\left(\mathrm{C}_{8}\right), 167.26\left(\mathrm{C}_{7}\right), 168.45\left(\mathrm{C}_{9}\right)$; MS: $\mathrm{m} / \mathrm{z}[596.10]^{+}$; Analysis calculated for $\mathrm{C}_{26} \mathrm{H}_{25} \mathrm{IN}_{6} \mathrm{O}_{3}: \mathrm{C}, 52.36 ; \mathrm{H}, 4.22 ; \mathrm{N}, 14.09$. Found: C, 52.23; H, 4.35; N, $14.17 \%$.

\section{2-[4-(2,3-Dichloro-phenyl)-piperazinyl-methyl]-3-[N-isonicotinamide-yl]-6-iodo- quinazoline-4-one (4l)}

Yield, 69\%; m.p. 232-234 ${ }^{\circ} \mathrm{C}$; FTIR (KBr) v: 3351 (NH), 3048 (C-H Aromatic), 2943 (C-H aliphatic), $1678(\mathrm{C}=\mathrm{O}$ of amide $), 1649(\mathrm{C}=\mathrm{O}$ of quinazolinone $), 1314(\mathrm{C}=\mathrm{N}), 1282(\mathrm{C}-\mathrm{N})$, $1260(\mathrm{~N}-\mathrm{N}), 526(\mathrm{C}-\mathrm{I}) \mathrm{cm}^{-1} ;{ }^{1} \mathrm{H}$ NMR $\left(400 \mathrm{MHz}, \mathrm{DMSO}-d_{6}\right) \delta \mathrm{ppm}: 1.85\left(\mathrm{~s}, 2 \mathrm{H}, \mathrm{CH}_{2}\right), 2.35-$ $2.57\left(\mathrm{~m}, 8 \mathrm{H}, 4 \times \mathrm{CH}_{2}\right), 6.47-8.14(\mathrm{~m}, 10 \mathrm{H}, \mathrm{Ar}-\mathrm{H}), 8.78$ (s, 1H, NH-CO); ${ }^{13} \mathrm{C}$ NMR $(100 \mathrm{MHz}$, DMSO- $\left.d_{6}\right) \delta$ ppm: $51.35\left(\mathrm{C}_{15}\right), 55.48\left(\mathrm{C}_{16}, \mathrm{C}_{19}\right), 57.30\left(\mathrm{C}_{17}, \mathrm{C}_{18}\right), 95.40\left(\mathrm{C}_{1}\right), 116.12-137.30$ $\left(\mathrm{C}_{3}-\mathrm{C}_{5}, \mathrm{C}_{10}-\mathrm{C}_{11}, \mathrm{C}_{20}-\mathrm{C}_{24}\right), 142.18\left(\mathrm{C}_{2}\right), 142.64\left(\mathrm{C}_{14}\right), 146.50\left(\mathrm{C}_{25}\right), 147.73\left(\mathrm{C}_{6}\right), 150.16$ $\left(\mathrm{C}_{12}-\mathrm{C}_{13}\right), 164.23\left(\mathrm{C}_{8}\right), 167.30\left(\mathrm{C}_{7}\right), 168.37\left(\mathrm{C}_{9}\right)$; MS: $\mathrm{m} / \mathrm{z}[634.01]^{+}$; Analysis calculated for $\mathrm{C}_{25} \mathrm{H}_{21} \mathrm{Cl}_{2} \mathrm{IN}_{6} \mathrm{O}_{2}$ : C, 47.27; H, 3.33; N, 13.23. Found: C, 47.38; H, 3.25; N, $13.34 \%$.

\section{In vitro evaluation of antibacterial and antifungal activities}

The MIC (Minimal Inhibition Concentration) of synthesized compounds was carried out by broth dilution method ${ }^{18}$. Serial dilutions were prepared for the purpose of the primary and secondary screening. Each synthesized drug was diluted obtaining $1000 \mu \mathrm{g} / \mathrm{mL}$ concentration, as a stock solution. In the primary screening $500,250,150$ and $125 \mu \mathrm{g} / \mathrm{mL}$ of the synthesized drugs was taken. The active synthesized drugs found in this primary screening were further tested in a second set of dilution against all microorganisms. The drugs found active in primary screening were similarly diluted to obtain $100,62.5,50,25,12.5,6.250,3.125$ and $1.5625 \mu \mathrm{g} / \mathrm{mL}$ concentrations. The highest dilution showing at least $99 \%$ inhibition is taken as MIC. The results of antibacterial and antifungal activity are summarized in Table 1.

Table 1. In vitro antibacterial activity (MIC $\mu \mathrm{g} / \mathrm{mL}$ ) of compounds $4 \mathbf{a}-\mathbf{I}$

\begin{tabular}{cccccccccc}
\hline Entry & $\mathrm{R}$ & $\mathrm{R}$ & E.c. & P.a. & Kl.p & S.t. & S.a. & S.p. & B.s. \\
\hline $\mathbf{4 a}$ & $-\mathrm{H}$ & $\mathrm{C}_{6} \mathrm{H}_{5}$ & 250 & 250 & 500 & 250 & 200 & 500 & 250 \\
$\mathbf{4 b}$ & $-\mathrm{H}$ & $\mathrm{C}_{6} \mathrm{H}_{5} \mathrm{CH}_{2}$ & 200 & 250 & 200 & 200 & 200 & 250 & 150 \\
$\mathbf{4 c}$ & $-\mathrm{H}$ & $\mathrm{CH}_{3}$ & 500 & 250 & 500 & 500 & 500 & 250 & 250 \\
$\mathbf{4 d}$ & $-\mathrm{H}$ & $2-\mathrm{OCH}_{3} \mathrm{C}_{6} \mathrm{H}_{5}$ & 125 & 150 & 150 & 200 & 200 & 150 & 200 \\
$\mathbf{4 e}$ & $-\mathrm{H}$ & $4-\mathrm{OCH}_{3} \mathrm{C}_{6} \mathrm{H}_{5}$ & 250 & 250 & 200 & 250 & 100 & 62.5 & 100 \\
$\mathbf{4 f}$ & $-\mathrm{H}$ & $2,3-\mathrm{diCl} \mathrm{C}_{6} \mathrm{H}_{5}$ & 62.5 & 100 & 62.5 & 62.5 & 100 & 125 & 100 \\
$\mathbf{4 g}$ & $-\mathrm{I}$ & $\mathrm{C}_{6} \mathrm{H}_{5}$ & 500 & 250 & 250 & 500 & 250 & 250 & 500 \\
$\mathbf{4 h}$ & $-\mathrm{I}$ & $\mathrm{C}_{6} \mathrm{H}_{5} \mathrm{CH}_{2}$ & 200 & 100 & 200 & 200 & 250 & 250 & 200 \\
$\mathbf{4 i}$ & $-\mathrm{I}$ & $\mathrm{CH}_{3}$ & 500 & 500 & 250 & 500 & 250 & 500 & 250 \\
$\mathbf{4 j}$ & $-\mathrm{I}$ & $2-\mathrm{OCH}_{3} \mathrm{C}_{6} \mathrm{H}_{5}$ & 150 & 200 & 150 & 250 & 250 & 150 & 200 \\
$\mathbf{4 k}$ & $-\mathrm{I}$ & $4-\mathrm{OCH}_{3} \mathrm{C}_{6} \mathrm{H}_{5}$ & 150 & 125 & 150 & 100 & 62.5 & 100 & 62.5 \\
$\mathbf{4 l}$ & $-\mathrm{I}$ & $2,3-\mathrm{diCl}_{6} \mathrm{C}_{6} \mathrm{H}_{5}$ & 125 & 62.5 & 150 & 150 & 200 & 125 & 150 \\
Gentamycin & - & - & 0.05 & 1 & 0.05 & 1 & 0.25 & 0.5 & - \\
Ampicilin & - & - & 100 & 100 & 100 & 100 & 250 & 100 & - \\
Chloramphenicol & - & - & 50 & 50 & 50 & 50 & 50 & 50 & - \\
Ciprofloxacin & - & - & 25 & 25 & 25 & 25 & 50 & 50 & - \\
Norfloxacin & - & - & 10 & 10 & 10 & 10 & 10 & 10 & - \\
\hline
\end{tabular}

E.c =, E. coli (MTCC 443); P.a= P. aeruginosa (MTCC 1688); Kl.p= Kl. pneumoniae (MTCC109); S.t. = S. typhi (MTCC98); S.a= S. aureus (MTCC 96); S.p=S. pyogenus (MTCC 442); B.s= B. subtilis (MTCC 441) 


\section{In vitro evaluation of antitubercular activity}

Drug susceptibility and determination of MIC of the test compounds against mycobacterium tuberculosis $H_{37} R v$ were performed by L. J. (Lowenstein and Jensen) MIC method ${ }^{19,20}$ for the measurement of MIC. Stock solutions of primary 1000, 500, $250 \mu \mathrm{g} / \mathrm{mL}$ and secondary $200,150,100,62.5,50,25,12.5,6.25$ and $3.25 \mu \mathrm{g} / \mathrm{mL}$ dilutions of each test compound in dimethylsulphoxide (DMSO) were added liquid L. J. Medium and then media were sterilized by inspissations method. A culture of mycobacterium tuberculosis $\mathrm{H}_{37} R v$ growing on L. J. Medium was harvested in $0.85 \%$ saline in bijou bottles. These tubes were then incubated at $37 \pm 1{ }^{\circ} \mathrm{C}$ for $24 \mathrm{~h}$ followed by streaking of mycobacterium tuberculosis $H_{37} R v$ $\left(5 \times 10^{4}\right.$ bacilli per tube). These tubes were then incubated at $37^{\circ} \mathrm{C}$. Growth of bacilli was seen after 12 days, 22 days and finally 28 days of incubation. Tubes having the compounds were compared with control tubes where medium alone was incubated with mycobacterium tuberculosis $H_{37} R v$. The concentration at which no development of colonies occurred or $<20$ colonies was taken as MIC concentration of test compound. The standard strain mycobacterium tuberculosis $H_{37} R v$ was tested with known drug rifampicin and isoniazid. The antitubercular data are shown in Table 2.

Table 2. In vitro antifungal activity (MIC $\mu \mathrm{g} / \mathrm{mL}$ ) of compounds 4a-1

\begin{tabular}{cccc}
\hline Entry & C.a. & A.n. & A.c. \\
\hline $\mathbf{4 a}$ & 1000 & 1000 & 1000 \\
$\mathbf{4 b}$ & 1000 & 500 & 1000 \\
$\mathbf{4 c}$ & 500 & 1000 & 500 \\
$\mathbf{4 d}$ & 500 & 250 & 500 \\
$\mathbf{4 e}$ & 250 & 200 & 200 \\
$\mathbf{4} \mathbf{f}$ & 500 & 500 & 500 \\
$\mathbf{4 g}$ & 250 & $>1000$ & $>1000$ \\
$\mathbf{4 h}$ & 1000 & $>1000$ & 1000 \\
$\mathbf{4 i}$ & 1000 & 500 & 1000 \\
$\mathbf{4 j}$ & 500 & 500 & 250 \\
$\mathbf{4 k}$ & 250 & 200 & 250 \\
$\mathbf{4 l}$ & 500 & 500 & 1000 \\
Nystatin & 100 & 100 & 100 \\
Greseofulvin & 500 & 100 & 100 \\
\hline
\end{tabular}

C.A=C. albicans (MTCC 227); A.N=A. niger (MTCC 282); A.C=A. clavatus (MTCC 1323).

\section{Results and Discussion}

The synthetic protocol used to synthesize the title compounds is outlined in Scheme 1. Compounds 4a-l was synthesized by using commercially available isonicotinic acid hydrazide. First, $N$-chloroacetyl substituted anthranilic acid $\mathbf{2 a / 2} \mathbf{b}$ was synthesized by the reaction of substituted anthranilic acid $\mathbf{1 a} / \mathbf{1} \mathbf{b}$ with chloroacetylchloride using dry benzene as solvent ${ }^{21}$. Further cyclisation ${ }^{21}$ of $\mathbf{2 a} / \mathbf{2 b}$ with isonicotinic acid hydrazide in the presence of dry $\mathrm{K}_{2} \mathrm{CO}_{3}$, yielded 2-chloromethyl-3-[ $N$-isonicotinamide-yl]-substituted-quinazolin-4-one $\mathbf{3 a} / \mathbf{3 b}$, which on further condensation with various substituted piperazine derivatives in the presence of anhydrous sodium carbonate gives desired compounds 4a-l. The structure of synthesized compounds was established by IR, $\left({ }^{1} \mathrm{H} \&{ }^{13} \mathrm{C}\right)-\mathrm{NMR}$, elemental analysis and mass spectral analysis. In the IR spectrum of $\mathbf{4 a - 1}$, the most characteristic absorption bands observed at 3336-3379 $\mathrm{cm}^{-1}(\mathrm{NH}), 2924-2959 \mathrm{~cm}^{-1}$ (C-H aliphatic) and 1271-1305 (C-N). In 
the ${ }^{1} \mathrm{H}-\mathrm{NMR}$ spectra of compounds $\mathbf{4 a - 1}$, NH peaks were observed as singlet at about $\delta 8.54$ $8.84 \mathrm{ppm}$ region. In addition, protons of piperazine were observed as multiplate at about $\delta$ 2.32-2.76 ppm region. All the other aromatic and aliphatic protons were observed at expected regions. From the ${ }^{13} \mathrm{C}-\mathrm{NMR}$ spectra it was observed that aliphatic carbon attached with piperazine ring appear at about $\delta 51.17-51.48 \mathrm{ppm}$ and carbon of piperazine ring was observed in the region of about $\delta 55.18-55.48$ and $\delta 57.24-57.83 \mathrm{ppm}$.<smiles>[R]c1ccc(N)c(C(=O)O)c1</smiles>

1

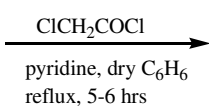

reflux, 5-6 hrs<smiles>[R]c1ccc(NC(=O)Cl)c(C(=O)O)c1</smiles>

2<smiles>NNC(=O)c1ccncc1</smiles><smiles>[R]c1ccc2nc(CCl)n(NC(=O)c3ccncc3)c(=O)c2c1</smiles>

anhy. $\mathrm{Na}_{2} \mathrm{CO}_{3}, \mathrm{C}_{2} \mathrm{H}_{5} \mathrm{OH}$ $8-9$ hrs

$\downarrow$<smiles>[R]c1ccc2nc(CN3CCN([R])CC3)n(NC(=O)c3ccncc3)c(=O)c2c1</smiles>

4a-l

Scheme 1. Synthesis of piperazinyl-quinazoline-1-one (4a-I)

From in vitro antibacterial and antifungal activity data, it is confirmed that compound $\mathbf{4 f}$ and $\mathbf{4 k}$ exhibited excellent activity against all tested gram negative strains and gram positive strains respectively while compounds $\mathbf{4 d}, \mathbf{4 j}$ and $\mathbf{4 k}$ displayed comparable activity against gram-negative strains. Other compounds are found to be moderate to good active against all antibacterial strain tested as compared to standard antibiotics. The in vitro antifungal activity data demonstrate that compounds $\mathbf{4 e}$ and $\mathbf{4 k}$ exhibited excellent antifungal activity against the fungal strain tested.

In general, the order of antibacterial activity of the substituent is 2, 3-dichloro phenyl $>$ 4-methoxy phenyl $>$ 2-methoxy phenyl $>$ benzyl $>\mathrm{H}>$ methyl and also 2,3-disubstituted $>$ 4-subsituted $>2$ - substituted is the order for better activity. Therefore, it can be inferred that presence of polar substituent imparts much towards antimicrobial potency ${ }^{12,13,22}$.

The encouraging results from the antibacterial and antifungal studies impelled us to go for preliminary screening of synthesized compounds against mycobacterium tuberculosis $H_{37} R v$. Among the newly synthesized compounds, compound $\mathbf{4 f}, \mathbf{4 h}$ and $\mathbf{4 l}$ produced highest efficacy and exhibited $>95 \%$ inhibition at a concentration of 50 and $62.5 \mu \mathrm{g} / \mathrm{mL}$ against mycobacterium tuberculosis $H_{37} R v$. Thus 2,3-dichloro substituent displayed relatively higher inhibitory activity in general. On the other hand with methoxy group $\mathbf{4 d}, \mathbf{4 e}, \mathbf{4 j}$ and $\mathbf{4 k}$ showed relatively low inhibitory activity against mycobacterium tuberculosis $H_{37} R v$. Thus, introduction of electron withdrawing substituent gives excellent antitubercular potency; this may be due to increased lipophilicity or with favorable steric hinderance ${ }^{12,13,22}$. 
Table 3. In vitro antitubercular activity of compounds 4a-I

\begin{tabular}{cccc}
\hline Entry & $\begin{array}{c}\text { M.Tuberculosis } H_{37} R v \\
(\mathrm{MIC} \mu \mathrm{g} / \mathrm{mL})(\text { MTCC } 200)\end{array}$ & \% Inhibition & Clogp $^{*}$ \\
\hline $\mathbf{4 a}$ & 250 & 85 & 0.61 \\
$\mathbf{4 b}$ & 100 & 95 & 1.47 \\
$\mathbf{4 c}$ & 250 & 88 & -0.24 \\
$\mathbf{4 d}$ & 250 & 90 & 0.63 \\
$\mathbf{4} \mathbf{4}$ & 150 & 91 & 0.63 \\
$\mathbf{4}$ & 50 & 96 & 2.15 \\
$\mathbf{4} \mathbf{g}$ & 250 & 89 & 1.80 \\
$\mathbf{4 h}$ & 62.5 & 96 & 2.65 \\
$\mathbf{4 i}$ & 250 & 34 & 0.93 \\
$\mathbf{4 j}$ & 250 & 87 & 1.82 \\
$\mathbf{4} \mathbf{k}$ & 200 & 90 & 1.82 \\
$\mathbf{4 l}$ & 62.5 & 95 & 3.33 \\
Rifampicin & 40 & 98 & 6.04 \\
Isoniazid & 0.20 & 99 & -0.60 \\
\hline
\end{tabular}

*Theoretical values of $\log P$ were calculated using commercially available chem draw program

\section{Conclusion}

Among all the newly synthesized compounds some compounds are showing good antituberculosis effect due to presence of three pharmacologically active nucleus viz. quinazolinone, piperazine and isoniazid. The importance of such work lies in the possibility that the new compounds obtained using such molecular hybridization might be a more efficacious against bacteria, mycobacteria and fungal infections, for which a thorough investigation regarding its structure activity relationship, toxicity and in vivo biological effects is essential, which is underway in our laboratory.

\section{Acknowledgement}

The authors wish to thank to Chairman - Charutar Vidya Mandal, Director-SICART \& Director - ARIBAS for providing necessary research facilities. We also extend our thanks to Mr. Priyakant R. Raval \& Dr. Kishor R. Desai, Director, Department of Chemistry, Uka Tarsadia University, Bardoli for research advice and encouragement.

\section{References}

1. a) Maity A, Mondal S, Paira R, Hazra A, Naskar S, Sahu K B, Saha P, Banerjee S and Mondal N B, Tetrahedron Lett., 2011, 52, 3033-3037; b) Akhaja T N and Raval J P, Chin Chem Lett., 2012, 23(4), 446-449; c) Akhaja T N and Raval J P, Eur J Med Chem., 2011, 46, 5573-5579.

2. $\quad$ Gupta S, Agarwal P K and Kundu B, Tetrahedron Lett., 2010, 51(14), 1887-1890.

3. Mhaske S B and Argade N P, Tetrahedron, 2006, 62, 9787-9826.

4. Jalani H B, Kaila C, Baraiya A B, Pandya A N, Sudarsanam V and Vasu K K, Tetrahedron Lett., 2010, 51, 5686-5689.

5. Hour M J, Huang L J, Kuo S C, Xia Y, Bastow K, Nakanishi Y, Hamel E and Lee K H, J Med Chem., 2000, 43, 4479-4487.

6. Askin D, Eng K K, Rossen K, Purick R M, Wells K M, Volante R P and Reider P J, Tetrahedron Lett., 1994, 35, 673-676. 
7. Kim B M, Evans B E, Gilbert K F, Hanifin C M, Vacca J P, Michelson S R, Darke P L, Zugay J A and Smini E A, Bioorg Med Chem Lett., 1995, 5(22), 2707-2712.

8. Foroumadi A, Emami S, Mansouri S, Javidnia A, Adeli N S, Shirazi F H and Shafiee A, Eur J Med Chem., 2007, 42, 985-992.

9. Cai M, Li Z, Fan F, Huang Q, Shao X and Song G, J Agric Food Chem., 2010, 58(5), 2624-2629.

10. (a) Singh K K, Joshi C S and Mathela C S, Ind J Chem., 2011, 50(2)B, 196-200;

(b) Shafiee A, Haddad Z M, Mohammadhosseini N, Khalafy J, Emami S, Moshafi M H., Sorkhi M and Foroumadi A, Daru J Pharm Sci., 2008, 16(3), 189-195.

11. Bali A, Sharma K, Bhalla A, Bala S, Reddy D, Singh A and Kumar A, Eur J Med Chem., 2010, 45, 2656-2662.

12. Todorovic A and Luevano H C, Peptides, 2005, 26, 2026-2036.

13. Kundu B, Curr Opin Drug Discov Develop., 2003, 6, 815-826.

14. Raval J P and Desai K R, Arkivoc, 2005, 13, 21-28.

15. Raval J P, Desai J T, Desai C K and Desai K R, Arkivoc, 2008, 12, 233-244.

16. Raval J P, Akhaja T N, Patel H V, Patel N H and Patel P S, Int J Pharm Res., 2009, 1, 62-71.

17. Raval J P, Patel H V, Patel P S, Patel N H and Desai K R, Asian J Res Chem., 2009, 2, 171-177.

18. Rattan A, Antimicrobials in Laboratory Medicine. Churchill B. I., Livingstone, New Delhi, 2000, 85.

19. Wermuth G, The Practice of Medicinal Chemistry, $2^{\text {nd }}$ Edition, Elsevier, 303.

20. Hagmann W K, J Med Chem., 2008, 51, 4359-4369.

21. Myangar K N and Raval J P, Med Chem Res., 2011; DOI: 10.1007/s00044-011-9808-9.

22. Malkia A, Murtomaki L, Urtti A and Kontturi K, Eur J Pharm Sci., 2004, 23(1), 13-47. 